\title{
Cyclodiphosphazanes as synthetic probes: P-C/P-N bond formation from the reaction with functionalized propargyl alcohols and $N$-hydroxy substrates
}

\author{
G GANGADHARARAO and K C KUMARA SWAMY* \\ School of Chemistry, University of Hyderabad, Hyderabad 500 046, India \\ e-mail:kckssc@uohyd.ac.in; kckssc@yahoo.com
}

MS received 29 July 2014; accepted 16 September 2014

\begin{abstract}
Phosphano-indoles were synthesized in a fairly straightforward route from the reaction of simple cyclodiphosphazanes $\left[\mathrm{XP}(\mu-\mathrm{N}-t-\mathrm{Bu})_{2} \mathrm{PY}\right][\mathrm{X}=\mathrm{Y}=\mathrm{NH}-t-\mathrm{Bu}(\mathbf{1 a}) ; \mathrm{X}=\mathrm{Y}=\mathrm{NH}-i-\mathrm{Pr}(\mathbf{1 b})]$ with $o-$ aminophenyl functionalized propargyl alcohols. The reaction occurs via an allene intermediate formed by $\mathrm{P}^{\mathrm{III}}$ $\mathrm{O}-\mathrm{C} \rightarrow \mathrm{P}^{\mathrm{V}}(\mathrm{O})-\mathrm{C}$ rearrangement, followed by cyclization utilizing the central allenic carbon and the $-\mathrm{NH}_{2}$ functionality. In a similar way, cyclodiphosphazanes $\left[\mathrm{XP}(\mu-\mathrm{N}-t-\mathrm{Bu})_{2} \mathrm{PY}\right][\mathrm{X}=\mathrm{Y}=\mathrm{Cl}(\mathbf{1 c}) ; \mathrm{X}=\mathrm{Cl}, \mathrm{Y}=\mathrm{NH}-t-\mathrm{Bu}$ (1d)] have been treated with $N$-hydroxy substrates to obtain novel $\mathrm{P}^{\mathrm{III}}-\mathrm{O}-\mathrm{N} \rightarrow \mathrm{P}^{\mathrm{V}}(\mathrm{O})-\mathrm{N}$ rearranged products. $\mathrm{X}$-ray structures of the four products, 2-(1-phenyl-ethyl)-3-[(t-Bu)NH)P( $\left.\mu-\mathrm{N}-t-\mathrm{Bu})_{2} \mathrm{P}(\mathrm{O})\right]$-indole [14], cis$\left\{\left[-\mathrm{C}(=\mathrm{O})-\mathrm{C}_{6} \mathrm{H}_{4}-\mathrm{C}(=\mathrm{O})-\right]-\mathrm{N}-\mathrm{P}(=\mathrm{O})-\mathrm{N}-t-\mathrm{Bu}\right\}_{2}\left[\right.$ cis-18], trans $-\left\{\left[-\mathrm{C}(=\mathrm{O})-\mathrm{C}_{6} \mathrm{H}_{4}-\mathrm{C}(=\mathrm{O})-\right]-\mathrm{N}-\mathrm{P}(=\mathrm{O})-\mathrm{N}-t-\mathrm{Bu}\right\}_{2}$ [trans-18] and cis-[(t-BuNH) $\left.\mathrm{P}(\mu-\mathrm{N}-t-\mathrm{Bu})_{2} \mathrm{P}(=\mathrm{O})-\mathrm{N}\left\{-\mathrm{C}(=\mathrm{O})-\mathrm{CH}_{2}-\mathrm{CH}_{2}-\mathrm{C}(=\mathrm{O})-\right\}\right][$ cis-19] are also reported.
\end{abstract}

Keywords. Cyclodiphosphazanes; cis-trans isomerism; allenes; indoles; rearrangement; propargyl alcohols; phosphano-heterocycles.

\section{Introduction}

Cyclodiphosph(III)azanes and their derivatives are well-established inorganic ring systems with alternate phosphorus and nitrogen atoms in their four-membered cyclic skeleton. ${ }^{1}$ Earlier, many studies in chemistry focused on the substitution reactions involved in the $\mathrm{P}-\mathrm{Cl}$ bond and the relative geometry of the substituents on phosphorus. ${ }^{1,2}$ Later, attention was shifted to utilize these ring systems as versatile ligands for metal complexes, ${ }^{3}$ precursors for macrocycles ${ }^{4}$ and synthetic probes to explore organic reaction pathways. ${ }^{5}$ The nucleophilic reactivity of cyclodiphosph(III)azanes towards alkenes, alkynes, allenylphosphonates and azo compounds has been gainfully employed in isolating the species analogous to the proposed intermediates in the well-known Mitsunobu reaction as well as 'umpolung addition' via phosphine activation of alkynes. ${ }^{5 \mathrm{c}, 6} \mathrm{In}$ the oxidative addition reactions, we have also reported rather unusual 'molecularly non-stoichiometric' crystals formed by this class of phosphorus compounds. ${ }^{7}$ The steric protection offered by the two-ring $\mathrm{N}-t-\mathrm{Bu}$ groups, convenient monitoring by ${ }^{31} \mathrm{P} \mathrm{NMR}$, and good crystallinity of the products in most cases make these

*For correspondence compounds wonderful precursors to probe organic reactions (chart 1).

In this study, although reaction of a normal alcohol or phenol with $[\mathrm{ClP}(\mu-\mathrm{NR})]_{2}$ leads to the substituted $\mathrm{P}^{\mathrm{III}}$ products, the reaction with propargyl alcohols affords tetracoordinate P-C bonded compounds cis-10 and trans-10 as shown in scheme 1a via a pseudo-Claisen-type rearrangement. ${ }^{8}$ These are potentially polymerizable phosphorus-based systems with alkene/alkyne substituents at two ends that would ultimately lead to polymer-terminated phosphonic acids as the end products. Allenes, and their subclass allenylphosphonates (phosphorylated allenes), are versatile precursors for diverse applications in organic synthesis. ${ }^{9-11}$ In this connection, recently, we reported the reactions of cyclodiphosphazanes with aryl substituted propargyl alcohols possessing $o$-nitro group that lead to rather previously unsuspected and unexpected products (e.g., 11, 12; scheme 1b). ${ }^{12}$ As part of our continued interest in studying the reactivity of $\mathrm{P}^{\mathrm{III}}$ compounds, in this study, we describe the first systematic studies on the reactivity of cyclophosphazanes with (a) $O$-amino-functionalized propargyl alcohols that afford phosphorus-based indoles and (b) $N$-hydroxy substrates that lead to $\mathrm{P}^{\mathrm{III}}-\mathrm{O}-\mathrm{N}$ $\rightarrow \mathrm{P}^{\mathrm{V}}(\mathrm{O})-\mathrm{N}$ rearranged products. The latter study is in continuation of our recent study concerning 


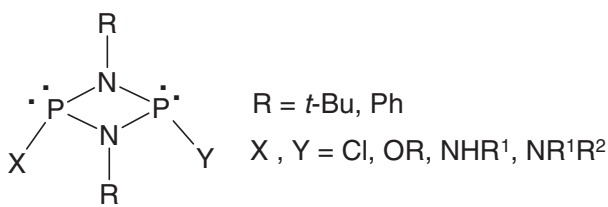

(1)<smiles>CCCCN(P(=S)(OC)OCC(C)(C)C)P(=S)(OCC(C)(C)C)N(C(C)(C)C)P(=S)(OCC(C)(C)C)N(C(C)(C)C)P(=S)(OCC(C)(C)C)OCC(C)(C)C</smiles>

2 (from ref. $4 b$ )

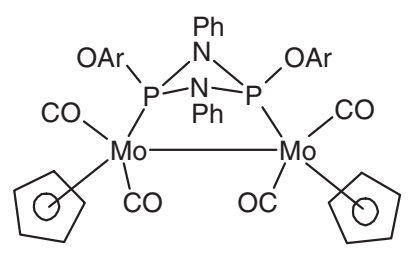

$\mathrm{Ar}=4-\mathrm{Me}-\mathrm{C}_{6} \mathrm{H}_{4}-$

5 (from ref. 2a)

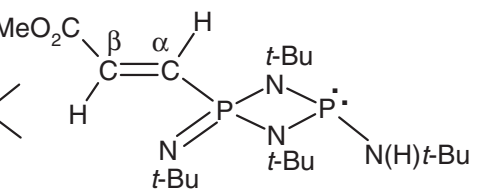

3 (from ref. 5c)

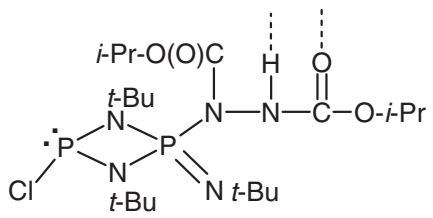

(Dimeric via hydrogen bonding) 4 (from ref. 5b)

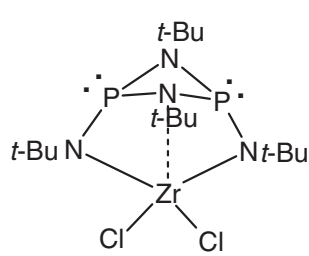

(N-connected complex) 6 (from ref. 3a)

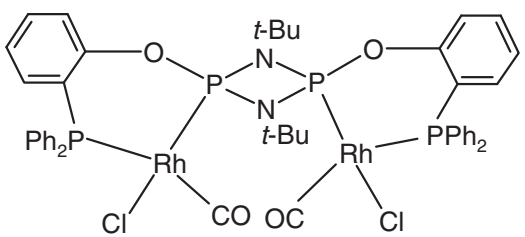

7 (from ref. 3e)

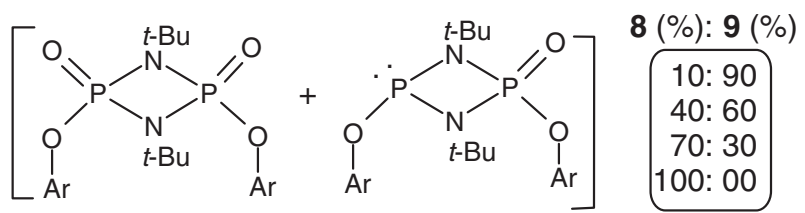

(8)

(9)

Molecularly nonstoichiometric crystals [ref. $7 \mathrm{~b}]$

Chart 1. Compounds 2-9 showing the utility of cyclodiphosphazanes.

the reaction of cyclophosphazanes with $N$-hydroxy succinimide. ${ }^{12,13}$

\section{Experimental}

All synthesis and manipulations were performed under nitrogen atmosphere unless stated otherwise. Chemicals/solvents were purified as required using standard procedures, ${ }^{14}$ unless otherwise noted. ${ }^{1} \mathrm{H},{ }^{13} \mathrm{C}$, and ${ }^{31} \mathrm{P}$ NMR spectra $\left({ }^{1} \mathrm{H} 400 \mathrm{MHz},{ }^{13} \mathrm{C} 100 \mathrm{MHz}\right.$, and ${ }^{31} \mathrm{P} 162 \mathrm{MHz}$ ) were recorded using a $\mathrm{CDCl}_{3}$ solution (unless stated otherwise) with shifts referenced to $\mathrm{SiMe}_{4}(\delta=0)$ or $85 \% \mathrm{H}_{3} \mathrm{PO}_{4}(\delta=0)$. Melting points were determined by using a hot stage melting point apparatus and were uncorrected. For TLC, glass microslides were coated with silica-gel-GF $\mathrm{GF}_{254}$ (mesh size $75 \mu$ ) and spots were identified using iodine or UV chamber as appropriate. For column chromatography, silica gel of 100-200 mesh size was used. Elemental analyses were carried out on a $\mathrm{CHN}$ analyzer. Mass spectra were recorded using LC-MS and HRMS
(ESI-TOF) equipment. Infrared spectra were recorded neat or by using $\mathrm{KBr}$ pellets on an FT/IR spectrometer.

\subsection{Synthesis of functionalized propargyl alcohols $(13 a-c)$-representative procedure for $13 c$}

A slightly modified literature procedure ${ }^{15}$ was used here. To a round bottomed flask $(50 \mathrm{~mL})$ equipped with 2-iodoaniline $(1.50 \mathrm{~g}, 6.80 \mathrm{mmol}), \mathrm{PdCl}_{2}(0.03 \mathrm{~g}$, $0.17 \mathrm{mmol}), \mathrm{PPh}_{3}(0.09 \mathrm{~g}, 0.34 \mathrm{mmol}), \mathrm{CuI}(0.06 \mathrm{~g}$, $0.34 \mathrm{mmol})$ and acetonitrile $(20 \mathrm{~mL})$, was added but-3-yn-2-ol (0.64 mL, $8.22 \mathrm{mmol})$ and $\mathrm{Et}_{3} \mathrm{~N}$ $(3.80 \mathrm{~mL}, 27.40 \mathrm{mmol})$ via syringe. Then, the mixture was stirred at room temperature $\left(25^{\circ} \mathrm{C}\right)$ for $7 \mathrm{~h}$ and the progress of the reaction monitored by TLC. Upon completion of the reaction, the crude mixture was filtered, the solid residue was washed with EtOAc, and the combined organic extract was concentrated under reduced pressure. Purification by column chromatography (hexane: ethyl acetate 4:1) gave the desired 4-(2aminophenyl)but-3-yn-2-ol (13c) as a gummy liquid. 


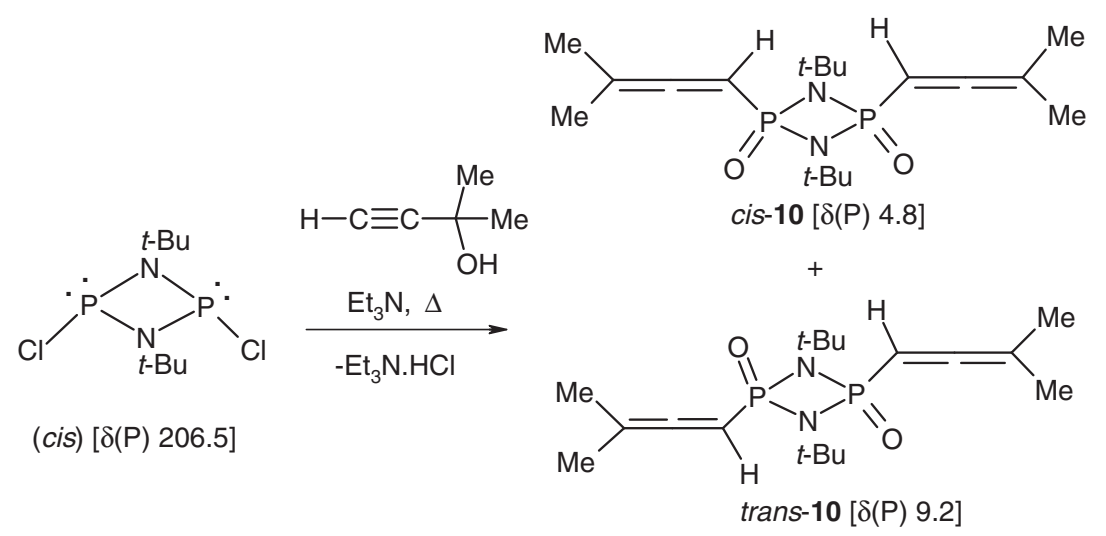

(a)

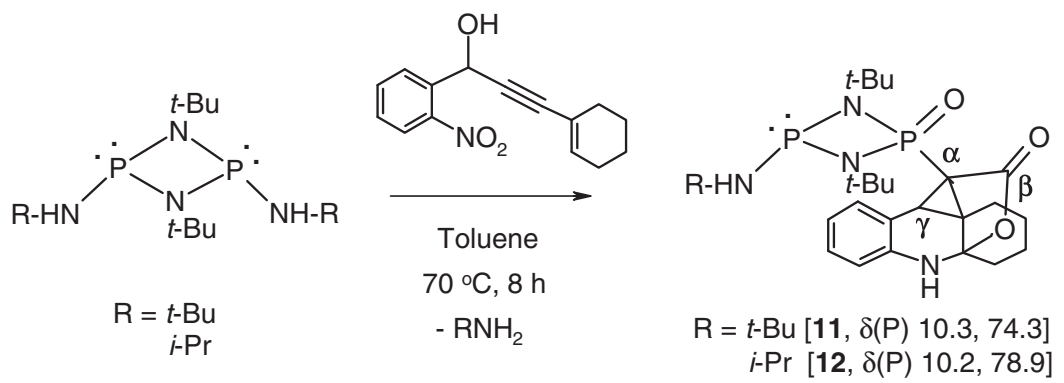

(b)

Scheme 1. Reaction of PIII-Cl precursors with propargyl alcohols leading to allene 10 or polycycles 11-12.

The known compounds $\mathbf{1 3 a}$ and $\mathbf{1 3} \mathbf{b}^{15}$ were prepared by following the same procedure.

2.1a Compound 13c: Yield: $1.36 \mathrm{~g}(91 \%) .{ }^{1} \mathrm{H}$ NMR $\left(400 \mathrm{MHz}, \mathrm{CDCl}_{3}, \mathrm{ppm}\right) \delta 7.27-6.66(\mathrm{~m}, 4 \mathrm{H}, \mathrm{Ar}-H)$, 4.78 (qrt, $1 \mathrm{H}, \mathrm{CHCH}_{3}$ ), 4.19 (br, $2 \mathrm{H}, \mathrm{NH}_{2}$ ), 3.41 (br, $1 \mathrm{H}, \mathrm{OH}), 1.55\left(\mathrm{~d}, J=6.4 \mathrm{~Hz}, 3 \mathrm{H}, \mathrm{CHCH}_{3}\right) .{ }^{13} \mathrm{C} \mathrm{NMR}$ $\left(100 \mathrm{MHz}, \mathrm{CDCl}_{3}, \mathrm{ppm}\right) \delta 147.8,132.2,129.7,118.0$, 114.5, 107.4 (Ar-C), $96.7(C \equiv \mathrm{C}), 80.5(\mathrm{C} \equiv \mathrm{C}), 58.7$ $\left(\mathrm{CH}\left(\mathrm{CH}_{3}\right)_{2}\right), 24.6\left(\mathrm{CHCH}_{3}\right)$. IR $\left(\mathrm{KBr}, \mathrm{cm}^{-1}\right) 3359$, 2980, 2931, 2219, 1616, 1490, 1457, 1364, 1314, 1161, 1101, 1079, 1029, 936, 744. LC-MS: $m / z, 162[\mathrm{M}+1]^{+}$. Anal. Calcd. for $\mathrm{C}_{10} \mathrm{H}_{11} \mathrm{NO}: \mathrm{C}, 74.51 ; \mathrm{H}, 6.88 ; \mathrm{N}, 8.69$. Found: C, 74.43; H, 6.75; N, 8.61.

\subsection{Synthesis of phosphazanyl indoles 14-17}

To a round bottomed flask $(25 \mathrm{~mL})$ equipped with cyclodiphosphazane $[(t-\mathrm{BuNH}) \mathrm{P}(\mu-\mathrm{N}-t-\mathrm{Bu})]_{2} \quad(\mathbf{1 a})^{5 \mathrm{c}}$ $(0.73 \mathrm{~g}, 2.1 \mathrm{mmol})$ and propargyl alcohol $(\mathbf{1 3 a})^{15}$ $(0.50 \mathrm{~g}, 2.1 \mathrm{mmol})$, was added dry toluene $(8 \mathrm{~mL})$. The mixture was stirred at $80^{\circ} \mathrm{C}$ for $4-5 \mathrm{~h}$, cooled to room temperature upon which a white solid material settled down. This was then filtered and washed with diethyl ether $(3 \times 10 \mathrm{~mL})$ to afford pure 2-(1-phenyl-ethyl),3$\left.[(t-\mathrm{Bu}) \mathrm{NH}) \mathrm{P}(\mu-\mathrm{N}-t-\mathrm{Bu})_{2} \mathrm{P}(\mathrm{O})\right]$-indole 14 as a white solid. Compounds 15-17 were prepared by following the same procedure.

2.2a Compound 14: Yield: $0.99 \mathrm{~g}(92 \%)$. mp: $252-$ $256^{\circ} \mathrm{C} .{ }^{1} \mathrm{H} \mathrm{NMR}\left(400 \mathrm{MHz}, \mathrm{CDCl}_{3}, \mathrm{ppm}\right) \delta 8.28(1 \mathrm{~s}$, $1 \mathrm{H}, \mathrm{NH}$ ), 7.67-7.12 (m, 9H, Ar- $H$ ), 5.88 (qrt, 1H, CH$\left.\mathrm{CH}_{3}\right), 3.32(\mathrm{~d}, J=7.6 \mathrm{~Hz}, 1 \mathrm{H}, \mathrm{NH}-t-\mathrm{Bu}), 1.74$ $\left(\mathrm{d}, J=7.6 \mathrm{~Hz}, 3 \mathrm{H}, \mathrm{CH}-\mathrm{CH}_{3}\right), 1.38(\mathrm{~d}, J=0.8 \mathrm{~Hz}$, $\left.9 \mathrm{H}, \mathrm{C}\left(\mathrm{CH}_{3}\right)_{3}\right), 1.26$ and $1.14\left(2 \mathrm{~s}, 18 \mathrm{H}, \mathrm{C}\left(\mathrm{CH}_{3}\right)_{3}\right)$. ${ }^{13} \mathrm{C}$ NMR $\left(100 \mathrm{MHz}, \mathrm{CDCl}_{3}, \mathrm{ppm}\right) \delta 153.1\left(\mathrm{~d},{ }^{2} J(\mathrm{P}-\right.$ C) $\sim 25.0 \mathrm{~Hz}, \mathrm{PCC}-\mathrm{NH}), 144.0,135.7,135.6,129.1$, 129.0, 128.6, 127.9, 126.5, 121.8, 120.6, 120.5, 120.4, 110.8, (Ar-C), $102.1\left(\mathrm{~d},{ }^{1} J(\mathrm{P}-\mathrm{C}) \sim 172.0 \mathrm{~Hz}, \mathrm{P}-C\right)$, $52.6\left(\mathrm{~d},{ }^{2} J(\mathrm{P}-\mathrm{C}) \sim 5.0 \mathrm{~Hz}, C\left(\mathrm{CH}_{3}\right)_{3}\right), 51.9\left(\mathrm{~d},{ }^{2} J(\mathrm{P}-\right.$ C) $\left.\sim 15.0 \mathrm{~Hz}, \mathrm{C}\left(\mathrm{CH}_{3}\right)_{3}\right), 35.9\left({ }^{\circ} 1 \mathrm{~s}, \mathrm{CH}-\mathrm{CH}_{3}\right), 32.9$ $\left(\mathrm{d},{ }^{3} J(\mathrm{P}-\mathrm{C})=10.0 \mathrm{~Hz}, \mathrm{C}\left(\mathrm{CH}_{3}\right)_{3}\right), 31.3\left(\mathrm{~d},{ }^{3} J(\mathrm{P}-\mathrm{C})\right.$ $\left.=5.0 \mathrm{~Hz}, \mathrm{C}\left(\mathrm{CH}_{3}\right)_{3}\right), 20.5\left(1 \mathrm{~s}, \mathrm{CH}-\mathrm{CH}_{3}\right) .{ }^{31} \mathrm{P} \mathrm{NMR}$ $\left(160 \mathrm{MHz}, \mathrm{CDCl}_{3}, \mathrm{ppm}\right) \delta 77.97\left(\mathrm{~d},{ }^{2} J(\mathrm{P}-\mathrm{P})=10.0 \mathrm{~Hz}\right.$, $(P-\mathrm{NH} t-\mathrm{Bu}), 10.67\left(\mathrm{~d},{ }^{2} J(\mathrm{P}-\mathrm{P})=10.0 \mathrm{~Hz}, P-\mathrm{C}\right) . \mathrm{IR}$ $\left(\mathrm{KBr}, \mathrm{cm}^{-1}\right)$ 3353, 3162, 3123, 3079, 2964, 2866, 1425, 1364, 1194, 1123, 1074, 888, 838. HRMS (ESI) calcd. for $\mathrm{C}_{28} \mathrm{H}_{42} \mathrm{~N}_{4} \mathrm{OP}_{2}(\mathrm{M}+\mathrm{H})^{+}$513.2913, found 513.2912.

2.2b Compound 15: This was prepared as a white solid by using $\left[(i-\mathrm{PrNH}) \mathrm{P}(\mu-\mathrm{N}-t-\mathrm{Bu}]_{2}(\mathbf{1 b})^{5 \mathrm{~d}}\right.$ 
$(0.42 \mathrm{~g}, 1.31 \mathrm{mmol})$ and propargyl alcohol 13a $(0.31 \mathrm{~g}$, $1.31 \mathrm{mmol}$ ) Yield: $0.529 \mathrm{~g}(81 \%)$. mp: $264-268^{\circ} \mathrm{C} .{ }^{1} \mathrm{H}$ NMR $\left(400 \mathrm{MHz}, \mathrm{CDCl}_{3}, \mathrm{ppm}\right) \delta 8.50(1 \mathrm{~s}, 1 \mathrm{H}, \mathrm{NH})$, 7.66-7.12 (m, 9H, Ar- $H$ ), 5.91 (qrt, $1 \mathrm{H}, \mathrm{CH}-\mathrm{CH}_{3}$ ), 3.65 (br, $\left.1 \mathrm{H}, \mathrm{NH}-\mathrm{CH}\left(\mathrm{CH}_{3}\right)_{2}\right), 3.01$ (br, $\left.1 \mathrm{H}, \mathrm{NH}-i-\mathrm{Pr}\right)$, $1.75\left(\mathrm{~d}, J=7.2 \mathrm{~Hz}, 3 \mathrm{H}, \mathrm{CH}-\mathrm{CH}_{3}\right), 1.24-1.22(\mathrm{~m}, 6 \mathrm{H}$, $\left.\mathrm{NH}-\mathrm{CH}\left(\mathrm{CH}_{3}\right)_{2}\right), 1.25$ and $1.14\left(2 \mathrm{~s}, 18 \mathrm{H}, \mathrm{C}\left(\mathrm{CH}_{3}\right)_{3}\right)$. ${ }^{13} \mathrm{C} \mathrm{NMR}\left(100 \mathrm{MHz}, \mathrm{CDCl}_{3}, \mathrm{ppm}\right) \delta 153.4\left(\mathrm{~d},{ }^{2} J(\mathrm{P}-\right.$ C) $\sim 25.0 \mathrm{~Hz}, \mathrm{PCC}-\mathrm{NH}), 144.1,135.9,135.7,129.1$, $128.9,128.5,128.3,127.9,126.4,121.7,120.6,120.5$, 120.3, $110.9(\mathrm{Ar}-C), 101.6\left(\mathrm{~d},{ }^{1} J(\mathrm{P}-\mathrm{C}) \sim 172.0 \mathrm{~Hz}\right.$, $\mathrm{P}-\mathrm{C}), 52.5\left(\mathrm{C}\left(\mathrm{CH}_{3}\right)_{3}\right), 44.9\left(1 \mathrm{~s}, \mathrm{NH}-\mathrm{CH}\left(\mathrm{CH}_{3}\right)_{2}\right), 35.9$ $\left(1 \mathrm{~s}, \mathrm{CH}-\mathrm{CH}_{3}\right), 31.2\left(1 \mathrm{~s}, \mathrm{C}\left(\mathrm{CH}_{3}\right)_{3}\right), 26.5(1 \mathrm{~s}, \mathrm{NH}-$ $\left.\mathrm{CH}\left(\mathrm{CH}_{3}\right)_{2}\right), 20.6\left(1 \mathrm{~s}, \mathrm{CH}-\mathrm{CH}_{3}\right) .{ }^{31} \mathrm{P}$ NMR $(160 \mathrm{MHz}$, $\left.\mathrm{CDCl}_{3}, \mathrm{ppm}\right) \delta 82.67$ (br, $\left.P-\mathrm{NH}-i-\mathrm{Pr}\right), 10.48(\mathrm{br}$, 1P, P-C). IR (KBr, cm $\left.{ }^{-1}\right) 3381,3167,3123,3090$, 3024, 2968, 2926, 2871, 1528, 1456, 1433, 1391, 1363, 1293, 1197, 1133, 1070, 891, 802. HRMS (ESI) calcd for $\mathrm{C}_{27} \mathrm{H}_{40} \mathrm{~N}_{4} \mathrm{OP}_{2}(\mathrm{M}+\mathrm{H})^{+}$499.2756, found 499.2757.

2.2c Compound 16: This was prepared as a white solid by using 1a $(0.64 \mathrm{~g}, 1.83 \mathrm{mmol})$ and propargyl alcohol 13b $(0.32 \mathrm{~g}, 1.83 \mathrm{mmol})$ Yield: $0.74 \mathrm{~g}(90 \%)$. mp: 296-298 ${ }^{\circ}$. ${ }^{1} \mathrm{H}$ NMR (400 MHz, $\mathrm{CDCl}_{3}+\mathrm{CD}_{3} \mathrm{OD}$, ppm) $\delta 7.78(\mathrm{br}, 1 \mathrm{H}, \mathrm{NH}), 7.60-7.10(\mathrm{~m}, 4 \mathrm{H}, \operatorname{Ar}-H)$, $4.37\left(\mathrm{~m}, 1 \mathrm{H}, \mathrm{CH}\left(\mathrm{CH}_{3}\right)_{2}, 3.51(\mathrm{br}, 1 \mathrm{H}, \mathrm{NH}-t-\mathrm{Bu})\right.$, $1.40\left(1 \mathrm{~s}, 9 \mathrm{H}, \mathrm{C}\left(\mathrm{CH}_{3}\right)_{3}\right), 1.35,\left(1 \mathrm{~s}, 6 \mathrm{H}, \mathrm{CH}\left(\mathrm{CH}_{3}\right)_{2}\right)$, $1.19\left(1 \mathrm{~s}, \quad 18 \mathrm{H}, \mathrm{C}\left(\mathrm{CH}_{3}\right)_{3}\right) .{ }^{13} \mathrm{C}$ NMR $(100 \mathrm{MHz}$, $\left.\mathrm{CDCl}_{3}+\mathrm{CD}_{3} \mathrm{OD}, \mathrm{ppm}\right) \delta 155.7\left(\mathrm{~d},{ }^{2} J(\mathrm{P}-\mathrm{C}) \sim 27.0 \mathrm{~Hz}\right.$, PCC-NH), 135.8, 128.3, 120.9, 119.5, 119.4, 110.3 $(\mathrm{Ar}-\mathrm{C}), 98.0\left(\mathrm{~d},{ }^{1} J(\mathrm{P}-\mathrm{C}) \sim 176.0 \mathrm{~Hz}, \mathrm{P}-\mathrm{C}\right), 52.0$ $\left(\mathrm{d},{ }^{2} J(\mathrm{P}-\mathrm{C}) \sim 6.0 \mathrm{~Hz}, C\left(\mathrm{CH}_{3}\right)_{3}\right), 51.0\left(\mathrm{~d},{ }^{2} J(\mathrm{P}-\mathrm{C})\right.$ $\left.\sim 14.0 \mathrm{~Hz}, C\left(\mathrm{CH}_{3}\right)_{3}\right), 32.0\left(\mathrm{~d},{ }^{3} J(\mathrm{P}-\mathrm{C})=10.0 \mathrm{~Hz}\right.$, $\left.\mathrm{C}\left(\mathrm{CH}_{3}\right)_{3}\right), 30.4\left(1 \mathrm{~s} \mathrm{C}\left(\mathrm{CH}_{3}\right)_{3}\right), 26.8\left(1 \mathrm{~s}, \mathrm{CH}\left(\mathrm{CH}_{3}\right)_{2}\right.$, 25.0 and $21.3\left(2 \mathrm{~s}, \mathrm{CH}\left(\mathrm{CH}_{3}\right)_{2}\right) .{ }^{31} \mathrm{P}$ NMR $(162 \mathrm{MHz}$, $\left.\mathrm{CDCl}_{3}+\mathrm{CD}_{3} \mathrm{OD}, \mathrm{ppm}\right) \delta 78.52\left(\mathrm{~d},{ }^{2} J(\mathrm{P}-\mathrm{P})=9.5 \mathrm{~Hz}\right.$, $(P-\mathrm{NH} t-\mathrm{Bu}), 13.48\left(\mathrm{~d},{ }^{2} J(\mathrm{P}-\mathrm{P})=9.5 \mathrm{~Hz}, P-\mathrm{C}\right) . \mathrm{IR}$ $\left(\mathrm{KBr}, \mathrm{cm}^{-1}\right) 3359,3140,2970,2860,1485,1458,1430$, 1359, 1288, 1211, 1129, 1058, 1030, 992, 878. HRMS (ESI) calcd. for $\mathrm{C}_{23} \mathrm{H}_{40} \mathrm{~N}_{4} \mathrm{OP}_{2}(\mathrm{M}+\mathrm{Na})^{+} 473.2575$, found 473.2577 .

2.2d Compound 17: This was prepared as a white solid by using 1a $(0.32 \mathrm{~g}, 0.91 \mathrm{mmol})$ and propargyl alcohol 13c (0.15 g, $0.91 \mathrm{mmol})$ Yield: $0.35 \mathrm{~g}(88 \%)$. mp: $278-280{ }^{\circ} \mathrm{C} .{ }^{1} \mathrm{H}$ NMR $\left(400 \mathrm{MHz}, \mathrm{CDCl}_{3}+\mathrm{CD}_{3} \mathrm{OD}\right.$, ppm) $\delta 7.75(1 \mathrm{~s}, 1 \mathrm{H}, \mathrm{NH}), 7.60-7.07(\mathrm{~m}, 4 \mathrm{H}, \mathrm{Ar}-$ $H), 4.09(\mathrm{~d}, J=7.6 \mathrm{~Hz}, 1 \mathrm{H}, \mathrm{NH}-t-\mathrm{Bu}), 3.22$ (qrt, $\left.2 \mathrm{H}, \mathrm{CH}_{2} \mathrm{CH}_{3}\right), 1.40\left(1 \mathrm{~s}, 9 \mathrm{H}, \mathrm{C}\left(\mathrm{CH}_{3}\right)_{3}\right), 1.35$ $\left(\mathrm{t}, 3 \mathrm{H}, \mathrm{CH}_{2} \mathrm{CH}_{3}\right), 1.19\left(1 \mathrm{~s}, 18 \mathrm{H}, \mathrm{C}\left(\mathrm{CH}_{3}\right)_{3}\right),{ }^{13} \mathrm{C}$
NMR $\left(100 \mathrm{MHz}, \mathrm{CDCl}_{3}+\mathrm{CD}_{3} \mathrm{OD}, \mathrm{ppm}\right) \delta 151.5$ (d, $\left.{ }^{2} J(\mathrm{P}-\mathrm{C}) \sim 25.0 \mathrm{~Hz}, \mathrm{PCC}-\mathrm{NH}\right), 135.5,128.5,128.3$, 120.7, 119.2, 119.0, $110.0(\mathrm{Ar}-C), 98.4\left(\mathrm{~d},{ }^{1} J(\mathrm{P}-\mathrm{C}) \sim\right.$ $177.0 \mathrm{~Hz}, \mathrm{P}-\mathrm{C}), 51.8\left(\mathrm{~d},{ }^{2} J(\mathrm{P}-\mathrm{C}) \sim 7.0 \mathrm{~Hz}, C\left(\mathrm{CH}_{3}\right)_{3}\right)$, $50.8\left(\mathrm{~d},{ }^{2} J(\mathrm{P}-\mathrm{C}) \sim 15.0 \mathrm{~Hz}, C\left(\mathrm{CH}_{3}\right)_{3}\right), 31.6\left(\mathrm{~d},{ }^{3} J(\mathrm{P}-\right.$ $\left.\mathrm{C})=10.0 \mathrm{~Hz}, \mathrm{C}\left(\mathrm{CH}_{3}\right)_{3}\right), 30.0\left(\mathrm{~d},{ }^{3} \mathrm{~J}(\mathrm{P}-\mathrm{C})=5.0 \mathrm{~Hz}\right.$, $\left.\mathrm{C}\left(\mathrm{CH}_{3}\right)_{3}\right), 19.7\left(1 \mathrm{~s}, \mathrm{CH}_{2} \mathrm{CH}_{3}\right), 12.7\left(1 \mathrm{~s}, \mathrm{CH}_{2} \mathrm{CH}_{3}\right)$. ${ }^{31} \mathrm{P}$ NMR $\left(162 \mathrm{MHz}, \mathrm{CDCl}_{3}+\mathrm{CD}_{3} \mathrm{OD}, \mathrm{ppm}\right) \delta 82.6(\mathrm{~d}$, ${ }^{2} J(\mathrm{P}-\mathrm{P})=9.2 \mathrm{~Hz},(P-\mathrm{NH} t-\mathrm{Bu}), 17.5\left(\mathrm{~d},{ }^{2} J(\mathrm{P}-\mathrm{P})=\right.$ 9.2 Hz, P-C). IR (KBr, cm $\left.{ }^{-1}\right) 3359,3090,2964,1458$, 1436, 1364, 1288, 1195, 1129, 1063, 992, 888. HRMS (ESI) calcd for $\mathrm{C}_{22} \mathrm{H}_{38} \mathrm{~N}_{4} \mathrm{OP}_{2}(\mathrm{M}+\mathrm{H})^{+}$437.2600, found 437.2601.

2.3 Synthesis of cis- and trans- $\left\{\left[-C(=O)-C_{6} H_{4}-\right.\right.$ $C(=O)-]-N-P(=O)-N-t-B u\}_{2}($ cis-18 and trans-1 8)

To a solution of $N$-hydroxy phthalimide $(0.494 \mathrm{~g}$, $3.02 \mathrm{mmol})$ and $\mathrm{NEt}_{3}(0.46 \mathrm{ml}, 3.33 \mathrm{mmol})$ in THF $(20 \mathrm{~mL})$ was added $[\mathrm{ClP}-\mathrm{N}(t-\mathrm{Bu})]_{2}(\mathbf{1 c})^{16}(0.417 \mathrm{~g}$, $1.51 \mathrm{mmol})$ dissolved in THF $(10 \mathrm{~mL})$ drop-wise at $0^{\circ} \mathrm{C}$ over a period of $15 \mathrm{~min}$. Then, the contents were brought to room temperature and stirred for $24 \mathrm{~h}$. After removal of amine hydrochloride (filtration) and solvent, the crude product was purified by column chromatography (hexane/EtOAc; 3:2) to afford cis-18 (white solid) followed by trans-18 (white solid). Cis-18 was crystallized from ethyl acetate and trans-18 was crystallized from dichloromethane.

2.3a Cis-18: Yield 0.115 g (14\%); mp 248-250 ${ }^{\circ} \mathrm{C}$; ${ }^{1} \mathrm{H}$ NMR $\left(400 \mathrm{MHz}, \mathrm{CDCl}_{3}\right) \delta 7.90-7.88(\mathrm{~m}, 4 \mathrm{H})$, 7.84-7.82 (m, 4H), 1.52 (s, 18H). ${ }^{13} \mathrm{C} \mathrm{NMR} \mathrm{(100} \mathrm{MHz,}$ $\left.\mathrm{CDCl}_{3}\right) \delta 166.6,135.3,132.2,124.3,58.0,30.1 .{ }^{31} \mathrm{P}$ NMR $\left(162 \mathrm{MHz}, \mathrm{CDCl}_{3}\right) \delta-17.40$. IR $\left(\mathrm{KBr}, \mathrm{cm}^{-1}\right)$ 3090, 3047, 2970, 2871, 1781, 1737, 1600, 1468, 1364, 1293, 1255, 1167, 1085, 1003, 904, 866. HRMS (ESI) calcd for $\mathrm{C}_{24} \mathrm{H}_{26} \mathrm{~N}_{4} \mathrm{O}_{6} \mathrm{P}_{2}(\mathrm{M}+\mathrm{H})^{+}$529.1407, found: 529.1407.

2.3b Trans-18: Yield $0.651 \mathrm{~g}$ (82\%); mp 250$252^{\circ} \mathrm{C} ;{ }^{1} \mathrm{H}$ NMR $\left(400 \mathrm{MHz}, \mathrm{CDCl}_{3}\right) \delta 8.04-8.02(\mathrm{~m}$, $4 \mathrm{H}), 7.88-7.86(\mathrm{~m}, 4 \mathrm{H}), 1.39(\mathrm{~s}, 18 \mathrm{H}) .{ }^{13} \mathrm{C} \mathrm{NMR}$ $\left(100 \mathrm{MHz}, \mathrm{CDCl}_{3}\right) \delta 166.2,135.5,132.3,124.7,56.8$, 30.0. ${ }^{31} \mathrm{P} \mathrm{NMR}\left(162 \mathrm{MHz}, \mathrm{CDCl}_{3}\right) \delta-20.36$. IR $(\mathrm{KBr}$, $\mathrm{cm}^{-1}$ ) 3083, 2976, 2928, 1794, 1748, 1717, 1645, 1599, 1468, 1373, 1300, 1265, 1192, 1148, 1051, 1015, 916. HRMS (ESI) calcd. for $\mathrm{C}_{24} \mathrm{H}_{26} \mathrm{~N}_{4} \mathrm{O}_{6} \mathrm{P}_{2}(\mathrm{M}+\mathrm{H})^{+}$ 529.1407, found: 529.1406 . 
2.4 Synthesis of cis- and trans- [(t-BuNH)P( $\mu-N-t$ $\left.\mathrm{Bu})_{2} \mathrm{P}(\mathrm{=O})-\mathrm{N}\left\{-\mathrm{C}(=\mathrm{O})-\mathrm{CH}_{2}-\mathrm{CH}_{2}-\mathrm{C}(=\mathrm{O})-\right\}\right]$ (cis-19 and trans-19)

The procedure was similar to that for compound $\mathbf{1 8}$ using $\left[(t-\mathrm{BuNH}) \mathrm{P}(\mu-\mathrm{N}-t-\mathrm{Bu})_{2} \mathrm{P}-\mathrm{Cl}\right] \quad(\mathbf{1 d})^{5 \mathrm{~b}} \quad(0.65 \mathrm{~g}$, $2.09 \mathrm{mmol})$ and $N$-hydroxysuccinimide $(0.24 \mathrm{~g}$, $2.09 \mathrm{mmol}$ ). After removing solvent, the residue was purified by column chromatography (hexane/EtOAc; 1:1) to afford pure cis-19 followed by trans-19.

2.4a Cis-19: Yield 0.572 g (70\%); mp 212-216 ${ }^{\circ} \mathrm{C}$; ${ }^{1} \mathrm{H}$ NMR $\left(400 \mathrm{MHz}, \mathrm{CDCl}_{3}\right) \delta 5.15(\mathrm{~d}, J=6.8 \mathrm{~Hz}$, 1H), $2.73(\mathrm{~s}, 4 \mathrm{H}),$,1.30 (s, 27H). ${ }^{13} \mathrm{C}$ NMR $(100 \mathrm{MHz}$, $\left.\mathrm{CDCl}_{3}\right) \delta 176.7,53.4(\mathrm{~d}, J \sim 8.0 \mathrm{~Hz}), 51.3(\mathrm{~d}, J \sim$ $17.0 \mathrm{~Hz}), 32.6(\mathrm{~d}, J=10.0 \mathrm{~Hz}), 31.0,29.5(\mathrm{~d}, J=$ $4.0 \mathrm{~Hz}) .{ }^{31} \mathrm{P}$ NMR $\left(162 \mathrm{MHz}, \mathrm{CDCl}_{3}\right) \delta 90.41,-10.47$. IR $\left(\mathrm{KBr}, \mathrm{cm}^{-1}\right) 3308,2971,2934,2872,1771,1721$, 1466, 1427, 1395, 1366, 1296, 1213, 1121, 1086, 1034, 1007, 930, 887, 853. HRMS (ESI) calcd. for $\mathrm{C}_{16} \mathrm{H}_{32} \mathrm{~N}_{4} \mathrm{O}_{3} \mathrm{P}_{2}(\mathrm{M}+\mathrm{H})^{+}$391.2029, found 391.2028.

2.4b Trans-19: Yield 0.151 g (19\%); mp 152$154^{\circ} \mathrm{C} ;{ }^{1} \mathrm{H}$ NMR $\left(400 \mathrm{MHz}, \mathrm{CDCl}_{3}\right) \delta 3.44(\mathrm{~d}, J=$ $4.8 \mathrm{~Hz}, 1 \mathrm{H}), 2.70(\mathrm{~s}, 4 \mathrm{H}), 1.33,1.30(2 \mathrm{~s}, 27 \mathrm{H}) .{ }^{13} \mathrm{C}$ NMR $\left(100 \mathrm{MHz}, \mathrm{CDCl}_{3}\right) \delta 172.3,53.1(\mathrm{~d}, J \sim 6.0 \mathrm{~Hz})$, $52.1(\mathrm{~d}, J \sim 16.0 \mathrm{~Hz}), 32.7(\mathrm{~d}, J=9.0 \mathrm{~Hz}), 31.0,25.5$. ${ }^{31} \mathrm{P}$ NMR $\left(162 \mathrm{MHz}, \mathrm{CDCl}_{3}\right) \delta 73.30(\mathrm{~d}, J=6.4 \mathrm{~Hz})$, $-11.15(\mathrm{~d}, J=6.4 \mathrm{~Hz})$. IR $\left(\mathrm{KBr}, \mathrm{cm}^{-1}\right) 3306,2973$, 2922, 2853, 1740, 1709, 1644, 1470, 1439, 1370, 1292, $1215,1103,999,918,885,851$. HRMS (ESI) calcd. for $\mathrm{C}_{16} \mathrm{H}_{32} \mathrm{~N}_{4} \mathrm{O}_{3} \mathrm{P}_{2}(\mathrm{M}+\mathrm{H})^{+}$391.2029, found: 391.2028.

2.5 Synthesis of cis [(t-BuNH)P( $\mu-N-t-B u)_{2} P(=O)$ $\left.N\left\{-C(=O)-C_{10} H_{6}-C(=O)-\right\}\right]($ cis-20)

Procedure was similar to that for $\mathbf{1 8}$ using cyclodiphosphazane $\left[(t-\mathrm{BuNH}) \mathrm{P}(\mu-\mathrm{N}-t-\mathrm{Bu})_{2} \mathrm{P}-\mathrm{Cl}\right] \quad(\mathbf{1 d})[0.35 \mathrm{~g}$, $1.12 \mathrm{mmol}], \quad N$-hydroxy-1,8-naphthalimide $(0.239 \mathrm{~g}$, $1.12 \mathrm{mmol})$ and $\mathrm{NEt}_{3}(0.16 \mathrm{~mL}, 1.12 \mathrm{mmol})$ The product was isolated by column chromatography (hexane/EtOAc; $1: 1)$ as a pale yellow solid 20. Yield: $0.375 \mathrm{~g}(68 \%)$; mp: $178-182^{\circ} \mathrm{C}$; ${ }^{1} \mathrm{H}$ NMR $(400 \mathrm{MHz}$, $\left.\mathrm{CDCl}_{3}, \mathrm{ppm}\right) \delta 8.66(\mathrm{~d}, J=7.6 \mathrm{~Hz}, 1 \mathrm{H}, \mathrm{Ar}-H), 8.59$ $(\mathrm{d}, J=7.2 \mathrm{~Hz}, 1 \mathrm{H}, \operatorname{Ar}-H), 8.28(\mathrm{~d}, J=3.2 \mathrm{~Hz}$, $1 \mathrm{H}, \operatorname{Ar}-H), 8.26(\mathrm{~d}, J=3.2 \mathrm{~Hz}, 1 \mathrm{H}, \operatorname{Ar}-H), 7.82(\mathrm{t}$, $1 \mathrm{H}, \operatorname{Ar}-H), 7.74(\mathrm{t}, 1 \mathrm{H}, \operatorname{Ar}-H), 6.66(\mathrm{~d}, J=7.6 \mathrm{~Hz}$, $1 \mathrm{H}, t-\mathrm{BuNH}), 1.41$ and $1.37\left(2 \mathrm{~s}, 27 \mathrm{H}, \mathrm{C}\left(\mathrm{CH}_{3}\right)_{3}\right) .{ }^{13} \mathrm{C}$ NMR $\left(100 \mathrm{MHz}, \mathrm{CDCl}_{3}, \mathrm{ppm}\right) \delta 173.0(1 \mathrm{~s}, C=\mathrm{O})$, $166.7(\mathrm{~d}, J=7.6 \mathrm{~Hz},(\mathrm{C}=\mathrm{O})-\mathrm{H}-\mathrm{N}), 135.4,134.6$, 132.7, 131.6, 131.1, 130.1, 127.8, 127.1, 123.8, 119.13, 119.1, $(\mathrm{Ar}-\mathrm{C}), 53.2\left(\mathrm{~d},{ }^{2} \mathrm{~J}(\mathrm{P}-\mathrm{C}) \sim 8.0 \mathrm{~Hz}, \mathrm{C}\left(\mathrm{CH}_{3}\right)_{3}\right)$, $51.9\left(\mathrm{~d},{ }^{2} J(\mathrm{P}-\mathrm{C}) \sim 18.0 \mathrm{~Hz}, C\left(\mathrm{CH}_{3}\right)_{3}\right), 32.2\left(\mathrm{~d},{ }^{3} J(\mathrm{P}-\right.$ $\left.\mathrm{C})=11.0 \mathrm{~Hz}, \mathrm{C}\left(\mathrm{CH}_{3}\right)_{3}\right), 31.2\left(1 \mathrm{~s}, \mathrm{C}\left(\mathrm{CH}_{3}\right)_{3}\right) .{ }^{31} \mathrm{P} \mathrm{NMR}$ $\left(160 \mathrm{MHz}, \mathrm{CDCl}_{3}, \mathrm{ppm}\right) \delta 82.10(1 \mathrm{~s}, 1 \mathrm{P},(P-\mathrm{NH} t-\mathrm{Bu})$, $-8.93(1 \mathrm{~s}, 1 \mathrm{P}, P-\mathrm{N})$. IR $\left(\mathrm{KBr}, \mathrm{cm}^{-1}\right) 3279,2969,2928$, 2866, 1676, 1632, 1588, 1559, 1510, 1468, 1372, 1316, 1208, 1142, 1092, 1038, 1011, 943, 880, 762. HRMS (ESI) calcd for $\mathrm{C}_{24} \mathrm{H}_{34} \mathrm{~N}_{4} \mathrm{O}_{3} \mathrm{P}_{2}(\mathrm{M}+\mathrm{H})^{+}$489.2185, found 489.2184 .

\section{$2.6 X$-ray structural analysis of $\mathbf{1 4}$, cis-18, trans-18,} and cis-19

Single crystal X-ray diffraction data for compounds $\mathbf{1 4}$ and trans-18 were collected on an OXFORD diffractometer and that for, cis-18, and cis-19 were collected on a Bruker AXS-SMART diffractometer using Mo $\mathrm{K}_{\alpha}$ $(\lambda=0.71073 \AA)$ radiation. The structures were solved and refined by standard methods. ${ }^{17}$ The data for compounds $\mathbf{1 4}$ and trans-18 were not good due to poor quality of the crystals, although the structures refined well. Full details of the X-ray structure solution and refinement as a CIF file are available as Supplementary Information.

2.6a Compound 14: colourless needles, $\mathrm{C}_{28} \mathrm{H}_{42} \mathrm{Br}_{2}$ $\mathrm{N}_{4} \mathrm{OP}_{2}, M=512.60$, Monoclinic, Space group $P 2_{1} / \mathrm{c}, a=13.4400(19), b=17.383(2), c=$ 13.2418(17) $\AA, \beta=104.546(13), V=2994.5(7) \AA^{3}$, $Z=4, \mu=0.171 \mathrm{~mm}^{-1}$, data/restraints/parameters: 4580/1/332, $\mathrm{R}$ indices $(\mathrm{I}>2 \sigma(\mathrm{I}))$ : $\mathrm{R} 1=0.1372, w \mathrm{R} 2$ (all data $)=0.2844$. The reflections were weak and the quality of the data was only moderate (hence an A alert was shown in checkcif), but good enough to fully refine the structure. Attempts to obtain better crystals were not successful. CCDC No. 1016234.

2.6b Cis-18: colourless block, $\mathrm{C}_{24} \mathrm{H}_{26} \mathrm{~N}_{4} \mathrm{O}_{6} \mathrm{P}_{2}, M=$ 528.43, Monoclinic, Space group $C 2 / \mathrm{c}, a=21.035$ (4), $b=10.233(2), c=15.523(3) \AA, \beta=128.87(3)$, $V=2601.4(9) \AA^{3}, Z=4, \mu=0.213 \mathrm{~mm}^{-1}$, data/ restraints/parameters: 2294/0/166, $\mathrm{R}$ indices (I > $2 \sigma(\mathrm{I})): \mathrm{R} 1=0.0411, w \mathrm{R} 2($ all data $)=0.1091 . \mathrm{CCDC}$ No. 927548.

2.6c Trans-18: colourless block, $\mathrm{C}_{25} \mathrm{H}_{28} \mathrm{~N}_{4} \mathrm{O}_{6} \mathrm{P}_{2} \mathrm{Cl}_{2}$, $M=613.35$, Triclinic, Space group $P \overline{1}, a=$ 7.9645(14), $b=9.0060(12), c=22.375(3) \AA, \alpha=$ 95.477(11), $\beta=93.033(13), \gamma=113.752(15), V=$ 1454.9(4) $\AA^{3}, Z=2, \mu=0.379 \mathrm{~mm}^{-1}$, data/restraints/ parameters: $5019 / 0 / 359, \mathrm{R}$ indices $(\mathrm{I}>2 \sigma(\mathrm{I}))$ : $\mathrm{R} 1=$ $0.1331, w \mathrm{R} 2$ (all data) $=0.2994$. CCDC No. 927549 . 
<smiles>[Y][P+]1N(C(C)(C)C)[PH+]([Y])[P+]1([Y])[R15]</smiles>
$\mathrm{X}, \mathrm{Y}=\mathrm{NH}-\mathrm{t}-\mathrm{Bu}$ $\mathrm{NH}-i-\mathrm{Pr}$ $\mathrm{Cl}$

$\mathrm{X}=\mathrm{Cl}, \mathrm{Y}=\mathrm{NH}-\mathrm{t}-\mathrm{Bu}$
(1a)
(1b)
(1c)
(1d)

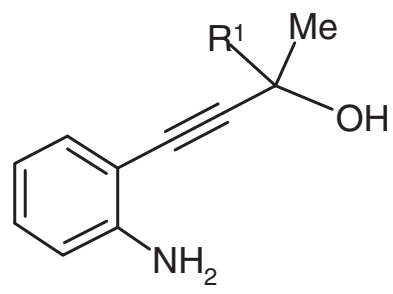

Chart 2. Cyclodiphosphazane precursors 1a-d and propargyl alcohols 13a-c used in this study.

The reflections were weak and the quality of the data was only moderate, but good enough to fully refine the structure.

2.6d cis-19: colourless block, $\mathrm{C}_{16} \mathrm{H}_{32} \mathrm{~N}_{4} \mathrm{O}_{3} \mathrm{P}_{2}$, $M=390.40$, Monoclinic, Space group $P 2_{1} / \mathrm{c}, a=$ 18.116(4), $b=10.276(2), c=11.554(2) \AA, \beta=$ 90.29(3), $V=2150.7(7) \AA^{3}, Z=4, \mu=0.223 \mathrm{~mm}^{-1}$, data/restraints/parameters: 3629/0/262, $\mathrm{R}$ indices (I > $2 \sigma(\mathrm{I})): \mathrm{R} 1=0.0740, w \mathrm{R} 2$ (all data) $=0.1505 . \mathrm{CCDC}$ No. 927550.

\section{Results and Discussion}

Cyclophosphazane precursors used in this study are $\left[\mathrm{XP}(\mu-\mathrm{N}-t-\mathrm{Bu})_{2} \mathrm{PY}\right]\left[\mathrm{X}=\mathrm{Y}=\mathrm{NH}-t-\mathrm{Bu}(\mathbf{1 a}),{ }^{5 \mathrm{c}} \mathrm{X}=\right.$ $\mathrm{Y}=\mathrm{NH}-i-\operatorname{Pr}(\mathbf{1 b}) ;{ }^{5 \mathrm{c}} \mathrm{X}=\mathrm{Y}=\mathrm{Cl}(\mathbf{1 c}) ;{ }^{16} \mathrm{X}=\mathrm{Cl}$,
$\mathrm{Y}=\mathrm{NH}-t-\mathrm{Bu}(\mathbf{1 d})] \cdot{ }^{5 \mathrm{~b}} o$-Amino functionalized propargyl alcohols (13a-c) were prepared from 2-iodo aniline and the corresponding propargyl alcohols by following standard procedures. ${ }^{15}$ These are shown in chart 2. We shall first discuss the reaction with propargyl alcohols followed by those with $N$-hydroxyphthalimide/ $N$ hydroxy succinimide/ $N$-hydroxy-1,8-naphthalimide.

\subsection{Reactivity of $[(R N H) P(\mu-N-t-B u)]_{2}[R=t-B u$ (1a), $i-\operatorname{Pr}(\mathbf{1 b})]$ towards functionalized propargylic alcohols 13a-c}

Initially, we treated the cyclodiphosphazanes $[(\mathrm{RNH}) \mathrm{P}(\mu-\mathrm{N}-t-\mathrm{Bu})]_{2}[\mathrm{R}=t$-Bu (1a), $i-\operatorname{Pr}(\mathbf{1 b})]$ with the aryl-substituted propargyl alcohol $\mathbf{1 3} \mathbf{a}^{15}$ possessing $o$-amino group. This led to cyclodiphosphazane-based substituted indoles $\mathbf{1 4}$ and $\mathbf{1 5}$ shown in scheme 2. These compounds are formed essentially as single products, but the solubility in organic solvents is rather poor. Here, the cyclodiphosphazane reacts with the propargyl alcohol forming the cyclodiphosphazane-based allene intermediate $\mathbf{I}^{8,12}$ that undergoes cyclization by the attack of $-\mathrm{NH}_{2}$ functionality on the $\beta$-carbon of the allene leading to the substituted cyclodiphosphazanebased indole. The IR spectra of $\mathbf{1 4}$ and $\mathbf{1 5}$ show the expected $v(\mathrm{NH})$ band in the region $3353-3381 \mathrm{~cm}^{-1}$. In the ${ }^{1} \mathrm{H}$ NMR spectrum of 14 , the $t$-BuN $H$ and indole$\mathrm{N} H$ peaks appear at $\delta 3.32$ and 8.28 , respectively. The corresponding signals for $\mathbf{1 5}$ appear at $\delta 3.01$ and 8.50, respectively. The ${ }^{31} \mathrm{P}$ NMR spectra of $\mathbf{1 4}$ shows two doublets at $\delta 10.7$ and 78.0 with ${ }^{2} J(\mathrm{PP})$ of $10.0 \mathrm{~Hz}$. In the case of $\mathbf{1 5}$, the broad signals appear at $\delta 10.5$

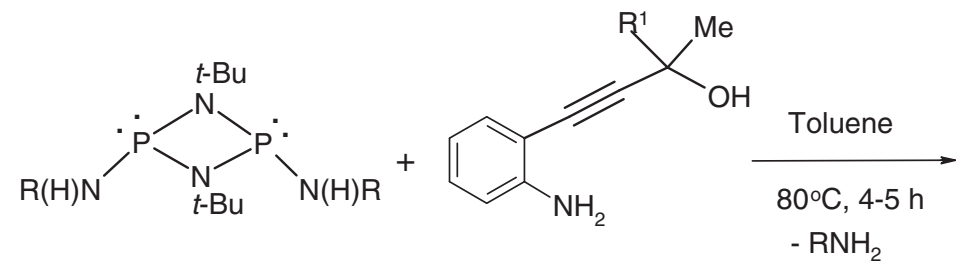

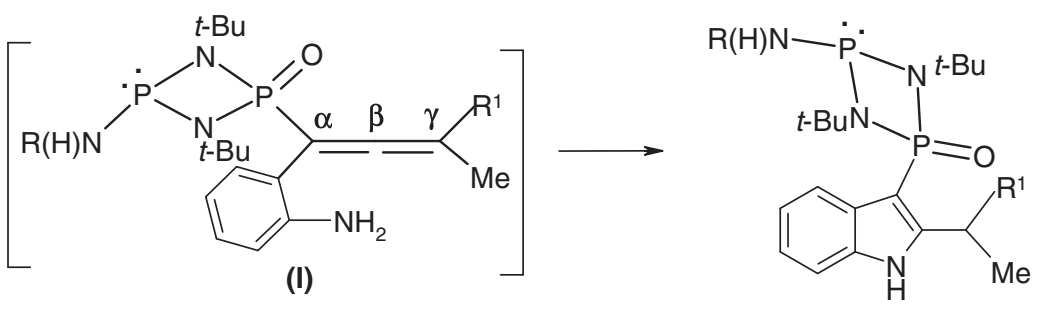

$$
\begin{aligned}
& \mathrm{R}=t-\mathrm{Bu}, \mathrm{R}^{1}=\mathrm{Ph}[1 \mathbf{1 4}, \delta(\mathrm{P}): 10.7,78.0 ; 92 \%, \mathrm{X} \text {-ray }] \\
& \mathrm{R}=i-\mathrm{Pr}, \mathrm{R}^{1}=\mathrm{Ph}[1 \mathbf{1 5}, \delta(\mathrm{P}): 10.5,82.7 ; 81 \%] \\
& \mathrm{R}=t-\mathrm{Bu}, \mathrm{R}^{1}=\mathrm{Me}[1 \mathbf{1 6}, \delta(\mathrm{P}): 13.5,78.5 ; 90 \%] \\
& \mathrm{R}=t-\mathrm{Bu}, \mathrm{R}^{1}=\mathrm{H} \quad[1 \mathbf{1 7} \delta(\mathrm{P}): 17.5,82.6 ; 88 \%]
\end{aligned}
$$

Scheme 2. Formation of cyclodiphosphazane-based indoles 14-17. 
and 82.7. In the ${ }^{13} \mathrm{C}$ NMR spectra, the ${ }^{1} J(\mathrm{P}-\mathrm{C})$ value of $172.0 \mathrm{~Hz}[\delta(\mathrm{C}) \sim 102]$ for $\mathbf{1 4}$ and $\mathbf{1 5}$ suggests that the corresponding phosphorus is connected to an $\mathrm{sp}^{2}$ carbon. ${ }^{5 \mathrm{c}}$ All these data are consistent with the structures shown in scheme 2. Compounds $\mathbf{1 6}$ and $\mathbf{1 7}$ were prepared similarly by using the cyclodiphosphazane (1a) and propargyl alcohols $\mathbf{1 3 b}$ and $\mathbf{1 3 c}$, respectively. The structure of compound $\mathbf{1 4}$ was further confirmed by single crystal X-ray diffraction (figure 1). Although the quality of X-ray data was only moderate, complete refinement could be effected and the formation of the $\mathrm{P}-\mathrm{C}$ bond as well as the indole ring can be clearly seen. Of the two isomers possible, the one in which indolyl group on one phosphorus is trans to the -NHR group on the second phosphorus is formed in the reaction.

\subsection{Reactivity of $[C l P-N(t-B u)]_{2}(1 c)$ and $[(t-B u N H)$} $\left.\mathrm{P}(\mu-\mathrm{N}-\mathrm{t}-\mathrm{Bu})_{2} \mathrm{P}-\mathrm{Cl}\right](\mathbf{1 d})$ towards $\mathrm{N}$-hydroxy derivatives

This part of the study initially was aimed at rationalizing the reaction of $\mathrm{P}^{\mathrm{III}}$ substrates with nitrofuntionalized propargyl alcohols from our group, wherein a $\mathrm{P}^{\mathrm{III}}-\mathrm{O}-\mathrm{N}$ to $\mathrm{P}^{\mathrm{V}}(\mathrm{O})-\mathrm{N}$ rearrangement was implicated. ${ }^{12}$ Here, we present a more detailed study on this aspect. Thus, we treated the cyclodiphosphazane [ClP-N $(t-\mathrm{Bu})]_{2}(\mathbf{1 c})$ with $N$-hydroxy phthalimide/ $\mathrm{Et}_{3} \mathrm{~N}$ and obtained the novel rearranged products cis-18 and trans-18 as shown in scheme 3. These compounds are formed by the substitution of a $-\mathrm{Cl}$ group by the $N$ hydroxyl reactant, followed by $\mathrm{P}^{\mathrm{III}}-\mathrm{O}-\mathrm{N} \rightarrow \mathrm{P}^{\mathrm{V}}(\mathrm{O})-\mathrm{N}$ rearrangement. To our knowledge, such reactions are

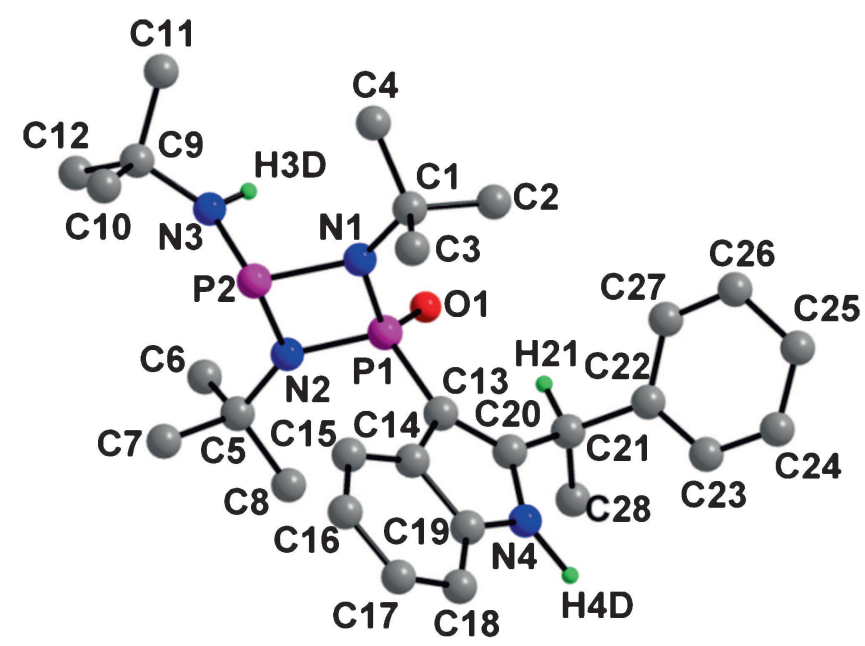

Figure 1. Molecular structure of compound 14. Hydrogen atoms (except $\mathrm{NH}$ and $\mathrm{CH}$ ) are omitted for clarity. Selected bond parameters: P1-C13 1.766(9), C13-C14 1.462(11), C13-C20 1.351(12), C20-C21 1.525(13), C20-N4 $1.384(11)(\AA)$.

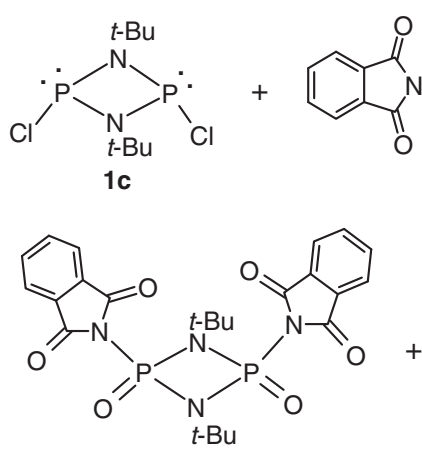

[cis-18, $\delta(P):-17.4,14 \%, X-$ ray $]$

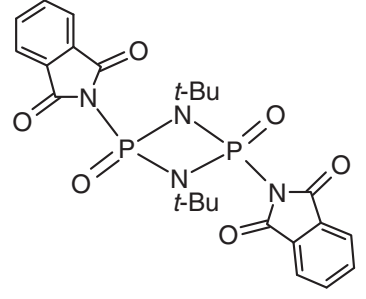

[trans-18, $\delta(P):-20.4,82 \%$, X-ray]
Scheme 3. Reaction of $N$-hydroxyphthalimide with 1c leading to $\mathrm{P}-\mathrm{N}$-substituted products cis-18 and trans-18.

never reported in cyclodiphosphazane chemistry prior to our study. In the reaction mixture, trans-18 is the major product and cis-18 is the minor product. The ${ }^{31} \mathrm{P}$ NMR spectra of cis-18 and trans-18 show signals at -17.4 , and -20.4 , respectively. This small difference $[\Delta \delta \sim 3 \mathrm{ppm}]$ in the ${ }^{31} \mathrm{P}$ NMR chemical shift values may be contrasted with the huge difference in the chemical shift values observed for cisand trans-amino-substituted cyclodiphosph(III)azanes $[\Delta \delta \sim 90 \mathrm{ppm}] .{ }^{18}$ The structures of these two compounds are further confirmed by X-ray crystallography (figures 2 and 3 ).

In an effort to probe whether there is any special preference for either of the isomers, cis or trans, we treated the chloro-substrate $\left[(t-\mathrm{BuNH}) \mathrm{P}(\mu-\mathrm{N}-t-\mathrm{Bu})_{2} \mathrm{P}-\right.$ Cl] (1d) with $N$-hydroxy succinimide. Here, we could isolate both the rearranged products cis-19 and trans19 (scheme 4; cis and trans of exocyclic P-N bonds

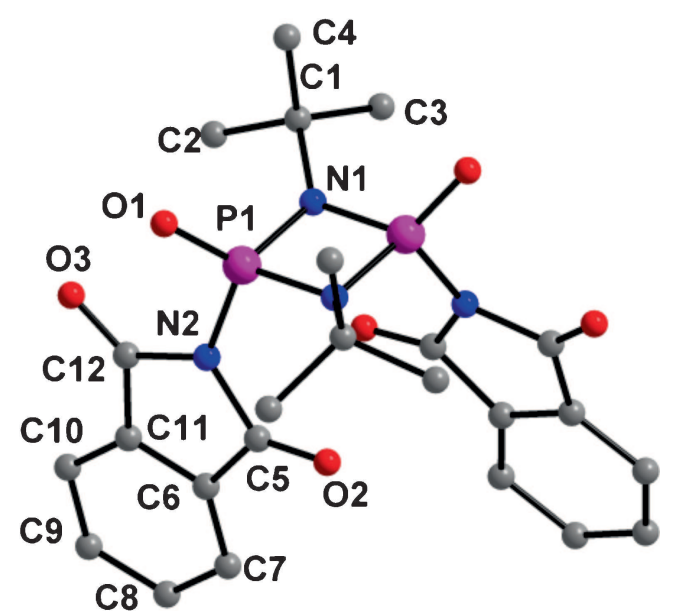

Figure 2. Molecular structure of cis-18. Hydrogen atoms are omitted for clarity. One identical half molecule present in the asymmetric unit. Selected bond distances: P1-N1 1.673(2), P1-N2 1.706(2), P1-O1 1.450(1) (̊). 


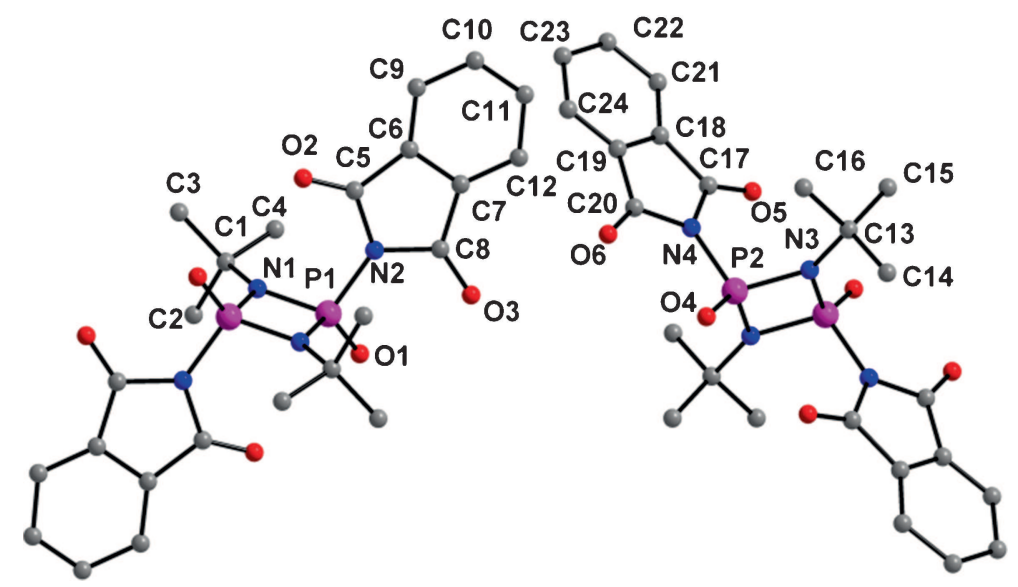

Figure 3. Molecular structure of compound trans-18 $1 / 2 \mathrm{CH} 2 \mathrm{Cl} 2$. Hydrogen atoms and the solvent molecule are omitted for clarity. Two essentially identical half molecules in the asymmetric unit are present. Selected bond parameters: P1-N1 1.643(6), P1-N2 1.708(7), P1-O1 1.451(6), P2-N3 1.679(7), P2-N4 1.713(7), P2-O4 1.443(6) (̊).

with respect to the cyclophosphazane ring) in yields of $70 \%$ and $19 \%$, respectively. This result shows that one isomer is predominant, but a direct comparison to cis-18 and trans-18 may not be appropriate because in the case of cis-19 and trans-19, only one phosphorus end has reacted with $N$-hydroxysuccinimide. The ${ }^{31} \mathrm{P}$ NMR spectra of $c i s-\mathbf{1 9}$ and trans-19 show two signals each at $[-10.5,90.4]$ and $[-11.2,73.3]$, respectively, which suggests that the two isomers are readily distinguishable in the $\mathrm{P}^{\mathrm{III}}$ region. We were able to obtain single crystals suitable for X-ray structure studies only in the case of cis-19. Compound cis-19 shows intra-molecular hydrogen bonding between $\mathrm{N}(3) \mathrm{H}$ and $\mathrm{C}(13)=\mathrm{O}(2)$ (figure 4). This feature could be a driving force for the predominance of isomer with this configuration.

We treated $N$-hydroxy-1,8-naphthalimide with cyclodiphosphazane $[\mathrm{ClP}-\mathrm{N}(t-\mathrm{Bu})]_{2}(\mathbf{1 c})$ also, but we

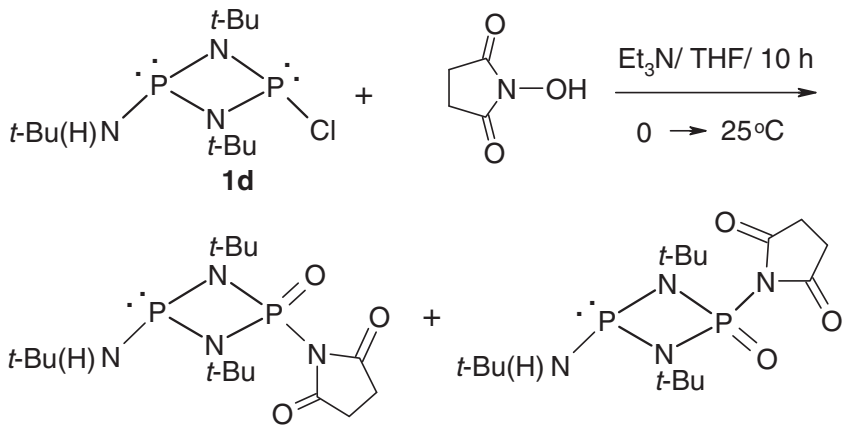

cis-19 $[\delta(\mathrm{P}):-10.5,90.4 ; 70 \%] \quad$ trans-19 $[\delta(\mathrm{P}):-11.2,73.3 ; 19 \%]$ (X-ray)

Scheme 4. Reaction of N-hydroxysuccinimide with 1d leading to P-N-subsitituted products cis-19 and trans-19. could not effect the reaction under these conditions However, the cyclodiphosphazane $[(t-\mathrm{BuNH}) \mathrm{P}(\mu-\mathrm{N}-$ $\left.t-\mathrm{Bu})_{2} \mathrm{P}-\mathrm{Cl}\right](\mathbf{1 d})$ afforded cis-20 shown in scheme 5 . The IR spectrum of cis-20 shows the $v(\mathrm{NH})$ band at $3279 \mathrm{~cm}^{-1}$. The ${ }^{31} \mathrm{P}$ NMR spectrum shows two signals at $\delta-8.9$ and $82.1\left[{ }^{2} J(\mathrm{PP})<3 \mathrm{~Hz}\right]$ as expected. The ${ }^{1} \mathrm{H}$ NMR spectrum of $c i s-\mathbf{2 0}$ shows the $t$-BuN $H$ peak at $\delta 6.70$. It may be noted that the $t$-BuN $H$ peak for cis-19 $[\delta 5.15]$ is much downfield compared to that for trans-19 [ $\delta 3.44]$. On this basis, we have assigned a cis-stereochemistry for compound $\mathbf{2 0}$. The assignment of cis-stereochemistry for $\mathbf{2 0}$ is also consistent with the fact that analogous reaction of $\mathbf{1 d}$ with $\mathrm{N}$ hydroxysuccinimide led to $c i s \mathbf{- 1 9}$ as the major product. The observation that $\mathbf{1 c}$ failed to react, but $\mathbf{1 d}$ reacted

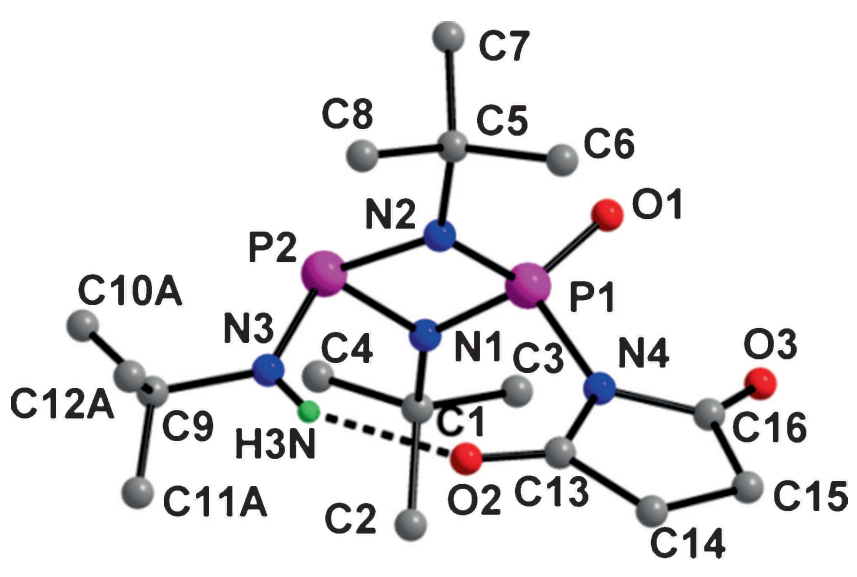

Figure 4. Molecular structure of compound cis-19. For the disordered t-butyl group at C9; only one position for the methyl groups is shown. Hydrogen atoms (except $\mathrm{NH}$ ) are omitted for clarity. Hydrogen bond parameters: N3H3N. . .O2 0.80(4) 2.17(4) 2.902(4) A, 154(4) ${ }^{\circ}$. 


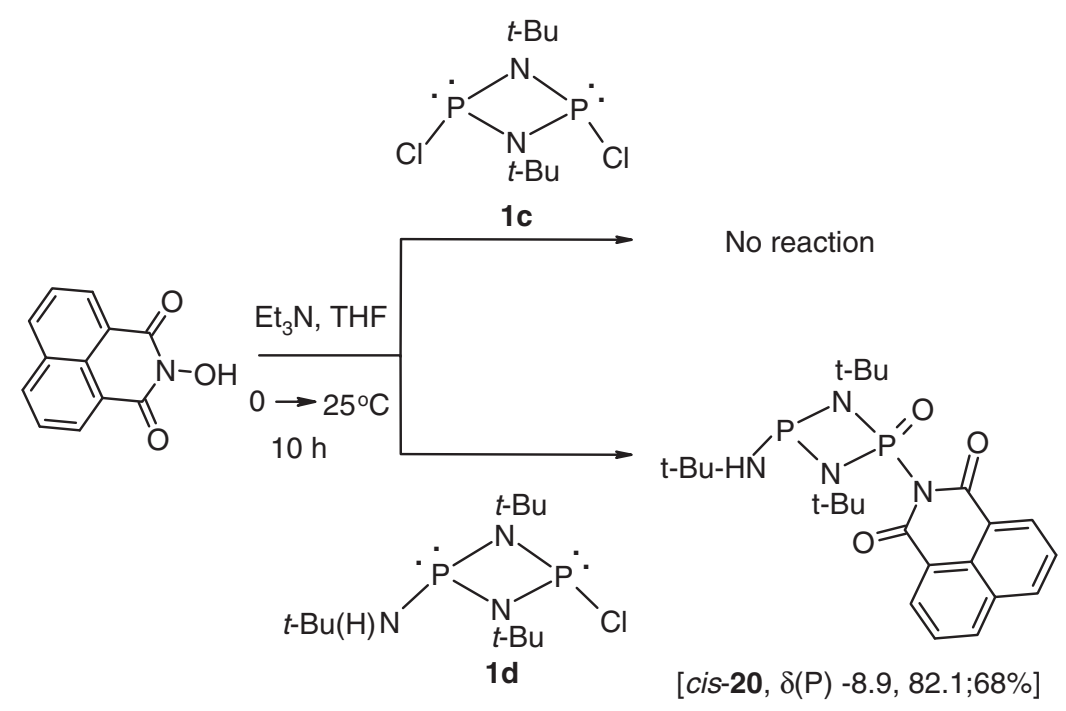

Scheme 5. Reaction of $N$-hydroxy-1,8-naphthalimide with $\mathbf{1 d}$ leading to P-N-subsitituted product $c i s-20$.

with $N$-hydroxy-1,8-naphthalimide may be linked to electronic factors. We have not probed this aspect further.

As regards the formation of products cis-18 and trans-18, involvement of intermediates II-III may be invoked (scheme 6). Rearrangement of the threemembered ring containing phosphonium betaine III will lead to compounds cis-18 and trans-18. In a similar manner, formation of compounds $\mathbf{1 9}$ and $\mathbf{2 0}$ may be rationalized. It may be noted that the phosphonium intermediate resulting from III bears some resemblance to the one proposed in Arbuzov rearrangement. However, no additional reagent similar to alkyl halide is used in our rearrangement. Formation of cis or trans isomers may be linked to the relative disposition of the substituents in the $\mathrm{P}^{\mathrm{III}}$ intermediate II.

\subsection{Brief comments on the structural aspects}

The P-N bond distances and selected angles in the compounds studied by X-ray crystallography along with analogous compounds 21-24 (chart 3) reported by us $^{12}$ are tabulated in table $1 . \mathrm{P}^{\mathrm{III}}-\mathrm{N}$ (ring) distances are always longer than the $\mathrm{P}^{\mathrm{V}}-\mathrm{N}$ (ring) distances. However, exocyclic $\mathrm{P}^{\mathrm{V}}-\mathrm{N}$ distances are longer than the corresponding $\mathrm{P}^{\mathrm{III}}-\mathrm{N}$ distances in all the cyclodiphosphazane derivatives. This feature suggests a competition for the bonding electrons. The endocyclic N-PII$\mathrm{N}$ angle is, in general, smaller than that of endocyclic $\mathrm{N}-\mathrm{P}^{\mathrm{V}}-\mathrm{N}$ angle probably due to (i) the lone pair of electrons on $\mathrm{P}^{\mathrm{III}}$, which tries to occupy more space, and (ii) the greater contribution from $\sim \mathrm{sp}^{3}$ hybridization at the $\mathrm{P}^{\mathrm{V}}$ centre. This feature could have forced the $\mathrm{P}^{\mathrm{III}}-\mathrm{N}$ (ring) distance to be longer. The $\mathrm{P}^{\mathrm{V}}-\mathrm{N}$ exocyclic distances are comparable to the apical P-N bonds in pentacoordinate phosphoranes ${ }^{19}$ and are slightly shorter than the expected P-N (single) bond distances based on Schomaker-Stevenson empirical expression $(1.77 \AA) .{ }^{20}$ The ring nitrogen atoms are essentially planar with the sum of the bond angles at nitrogen close to $360^{\circ}$. The four-membered $\mathrm{P}_{2} \mathrm{~N}_{2}$ ring in compounds cis-18, trans-18 and $\mathbf{2 3}$ is planar, but in other cases, deviations do occur to an extent of $0.03-0.06 \AA$ from the mean plane.

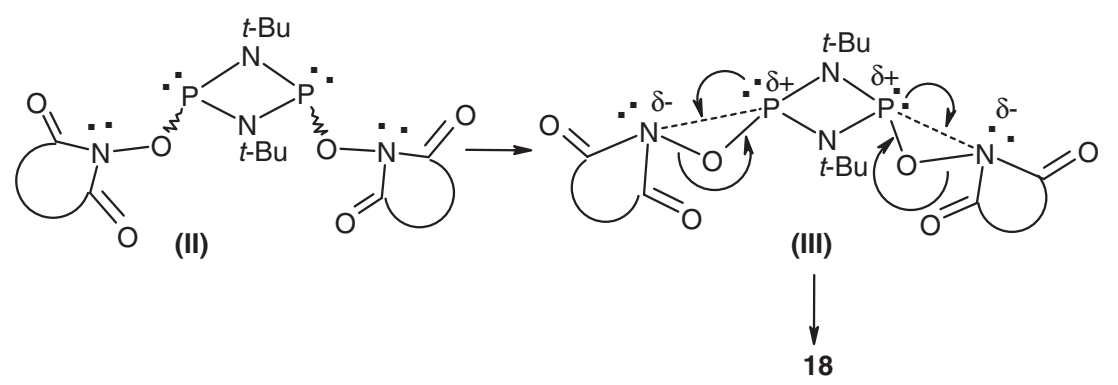

Scheme 6. Possible pathway for the $\mathrm{P}^{\mathrm{III}}-\mathrm{O}-\mathrm{N} \rightarrow \mathrm{P}^{\mathrm{V}}(\mathrm{O})-\mathrm{N}$ rearrangement. 


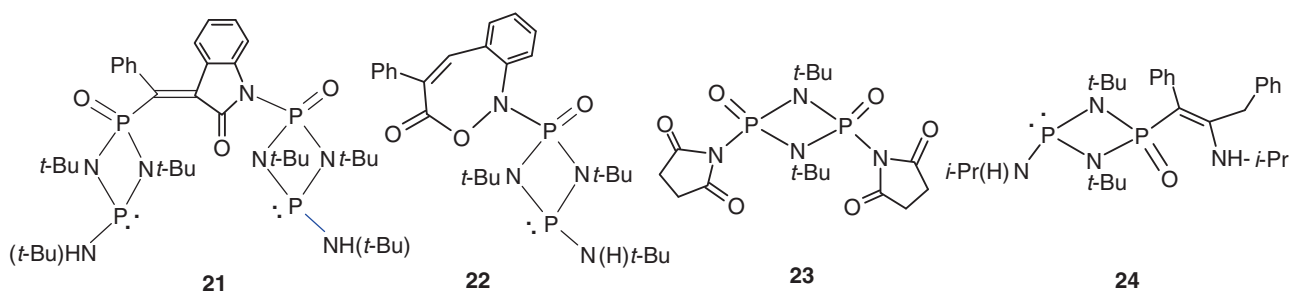

Chart 3. Selected compounds from ref. ${ }^{12}$.

Table 1. Comparison of P-N bond parameters in cyclodiphosphazane derivatives.

\begin{tabular}{|c|c|c|c|c|c|c|}
\hline Compound & $\mathrm{P}^{\mathrm{V}}-\mathrm{N}$ (ring) & $\mathrm{P}^{\mathrm{III}}-\mathrm{N}$ (ring) & $\mathrm{P}^{\mathrm{V}}-\mathrm{N}(\mathrm{exo})$ & $\mathrm{P}^{\mathrm{III}}-\mathrm{N}(\mathrm{exo})$ & $\mathrm{N}-\mathrm{P}^{\mathrm{V}}-\mathrm{N}($ ring $)$ & $\mathrm{N}-\mathrm{P}^{\mathrm{III}}-\mathrm{P}($ ring $)$ \\
\hline \multirow[t]{4}{*}{ 12. $1 / 2 \mathrm{H}_{2} \mathrm{O}\left(\right.$ ref. $\left.^{12}\right)$} & $1.649(4)$ & $1.743(5)$ & & $1.662(5)$ & $85.2(2)$ & $79.1(2)$ \\
\hline & $1.649(5)$ & $1.760(4)$ & & $1.654(5)$ & $86.0(2)$ & $79.0(2)$ \\
\hline & $1.643(5)$ & $1.752(4)$ & & & & \\
\hline & $1.639(4)$ & $1.767(5)$ & & & & \\
\hline \multirow{2}{*}{14 (Present study) } & $1.652(7)$ & $1.749(9)$ & - & $1.642(9)$ & $85.2(4)$ & $79.2(4)$ \\
\hline & $1.636(8)$ & $1.744(8)$ & & & & \\
\hline \multirow[t]{2}{*}{ Cis-18 (Present study) } & $1.673(2)$ & & $1.706(2)$ & & $85.3(1)$ & \\
\hline & $1.655(2)$ & & & & & \\
\hline \multirow[t]{4}{*}{ Trans-18 $\cdot 1 / 2 \mathrm{CH}_{2} \mathrm{Cl}_{2}$ (Present study) } & $1.643(6)$ & $1.708(7)$ & & & 84.5(3), & \\
\hline & $1.672(6)$ & $1.713(7)$ & & & $86.1(4)$ & \\
\hline & $1.679(7)$ & & & & & \\
\hline & $1.670(7)$ & & & & & \\
\hline \multirow[t]{2}{*}{ cis-19 } & $1.626(3)$ & $1.750(3)$ & $1.738(3)$ & $1.638(3)$ & $86.5(2)$ & $79.3(1)$ \\
\hline & $1.631(3)$ & $1.747(3)$ & & & & \\
\hline \multirow[t]{4}{*}{21 (ref. $^{12}$ ) } & $1.644(3)$ & $1.750(3)$ & $1.716(3)$ & $1.663(3)$ & $85.7(1)$ & $78.9(1)$ \\
\hline & $1.642(3)$ & $1.763(3)$ & & $1.646(3)$ & $86.6(1)$ & $79.0(1)$ \\
\hline & $1.634(3)$ & $1.769(3)$ & & & & \\
\hline & $1.631(3)$ & $1.752(3)$ & & & & \\
\hline \multirow[t]{2}{*}{ 22. $\mathrm{PhCH}_{3}$ (ref. ${ }^{12}$ ) } & $1.652(4)$ & $1.736(4)$ & $1.697(3)$ & $1.642(4)$ & $85.3(2)$ & $79.5(2)$ \\
\hline & $1.639(3)$ & $1.736(4)$ & & & & \\
\hline \multirow[t]{2}{*}{23 (ref. $^{12}$ ) } & $1.667(2)$ & $1.705(2)$ & & & $85.5(1)$ & - \\
\hline & $1.650(2)$ & & & & & \\
\hline \multirow[t]{2}{*}{$\mathbf{2 4} \cdot 1 / 2 \mathrm{H}_{2} \mathrm{O}$ (ref. ${ }^{12}$ ) } & $1.658(2)$ & $1.743(2)$ & & $1.653(2)$ & $84.9(1)$ & $79.7(1)$ \\
\hline & $1.652(2)$ & $1.741(2)$ & & & & \\
\hline
\end{tabular}

\section{Summary}

We have developed a straightforward route to synthesize cyclophosphazane-based indoles starting from ortho-amino functionalized propargyl alcohols via allene intermediates. We have isolated these indoles directly from the reaction mixture without performing column chromatography. We have also synthesized novel P-N bonded compounds using cyclodiphosphazanes and $N$-hydroxy substrates which involve a rearrangement from $\mathrm{P}^{\mathrm{III}}-\mathrm{O}-\mathrm{N}$ to $\mathrm{P}^{\mathrm{V}}(\mathrm{O})-\mathrm{N}$, a feature previously not reported in cyclophosphazane chemistry.

\section{Supplementary Information}

The electronic supporting information (CIF file containing the details of crystal structures of compounds reported in this work) and selected NMR data (figures $\mathrm{S} 1-\mathrm{S} 4)$ can be seen in www.ias.ac.in/chemsci.

\section{Acknowledgements}

We thank the Department of Science and Technology (DST, New Delhi) for financial support and for setting up of the National Single Crystal Diffractometer Facility at the University of Hyderabad. We also thank UPE program of the University Grants Commission (UGC, New Delhi) for equipment. KCK thanks DST for J. C. Bose fellowship and GGR thanks CSIR for a fellowship.

\section{References}

1. (a) Keat R 1982 Top. Curr. Chem. 102 89; (b) Kumaravel S S, Krishnamurthy S S, Cameron T S and Linden A 
1988 Inorg. Chem. 27 4546; (c) Stahl L 2000 Coord. Chem. Rev. 210 203; (d) Briand G G, Chivers T and Krahn M 2002 Coord. Chem. Rev. 233-234 237

2. (a) Reddy V S, Krishnamurthy S S and Nethaji M 1994 J. Chem. Soc., Dalton Trans. 2661 and references cited therein; (b) Vijjulatha M, Kumara Swamy K C, Vittal J J and Koh L L 1999 Polyhedron 182249 and references cited therein

3. (a) Grocholl L, Stahl L and Staples R J 1997 Chem. Commun. 1465; (b) Moser D, Grocholl L, Stahl L and Staples R J 2003 J. Chem. Soc., Dalton Trans. 1402; (c) Briand G G, Chivers T, Parvez M and Schatte G 2003 Inorg. Chem. 42 525; (d) Balakrishna M S, Venkateswaran R and Mague J T 2009 Inorg. Chem. 48 1398; (e) Balakrishna M S, Venkateswaran R and Mague J T 2010 Dalton Trans. 39, 11149; (f) Balakrishna M S, Suresh D, Rai A, Mague J T and Panda D 2010 Inorg. Chem. 49, 8790; (g) Roth T, Wadepohl H, Wright D S, Gade L H 2013 Chem. -Eur. J. 19 13823; (h) Balakrishna M S, Suresh D, Ananthnag G S and Mague J T 2014 Dalton Trans. 43, 8835

4. (a) Gonce F, Caminade A M, Boutonnet F and Majoral J P 1992 J. Org. Chem. 57 970; (b) Kommana P and Kumara Swamy K C 2000 Inorg. Chem. 39 4384; (c) Kommana P, Pavan Kumar K V P and Kumara Swamy K C 2003 Indian J. Chem. 42A 2371; (e) Garcia F, Goodman J M, Kowenicki R A, McPartlin M, Riera L, Silva M A, Wirsing A and Wright D S 2005 Dalton Trans. 1764; (d) Dodds F, García F, Kowenicki R A, McPartlin M, Steiner A and Wright D S 2005 Chem. Commun. 3733; (e) Chandrasekaran P, Mague J T and Balakrishna M S 2009 Dalton Trans. 5478

5. a) Satish Kumar N, Praveen Kumar K, Pavan Kumar K V P, Kommana P, Vittal J J and Kumara Swamy K C 2004 J. Org. Chem. 69 1881; (b) Kumara Swamy K C, Praveen Kumar K and Bhuvan Kumar N N 2006 J. Org. Chem. 71 1002; (c) Bhuvan Kumar N N, Chakravarty M and Kumara Swamy K C 2006 New J. Chem. 30, 1614; (d) Kumara Swamy K C, Gangadhararao G, Rama Suresh R, Bhuvan Kumar N N and Chakravarty M 2010 J. Organomet. Chem. 6951042

6. (a) Lu X, Zhang C and Xu Z 2001 Acc. Chem. Res. 34, 535; (b) Kumara Swamy K C, Bhuvan Kumar N N, Balaraman E and Pavan Kumar K V P 2009 Chem. Rev. 1092551

7. (a) Chakravarty M, Kommana P and Kumara Swamy K C 2005 Chem. Commun. 5396. (b) Chakravarty M, Rama Suresh R and Kumara Swamy K C 2007 Inorg. Chem. 469819

8. Bhuvan Kumar N N and Kumara Swamy K C 2007 Polyhedron 26883
9. (a) Krause $\mathrm{N}$ and Hashmi A S K (Editors) Modern Allene Chemistry 2004 Wiley-VCH: Weinheim, pp. 760-787; (b) Ma S 2009 Acc. Chem. Res. 42 1679; (c) Alcaide B Almendros P and Aragoncillo C 2010 Chem. Soc. Rev. 39 783; (d) Back T G Clary K N and Gao D 2010 Chem. Rev. 110 4498; (e) Krause N and Winter C 2011 Chem. Rev. 111 1994; (f) Yu S and Ma S 2012 Angew. Chem. Int. Ed. 513074

10. Phosphorylated allene synthesis: (a) Bhuvan Kumar N N, Chakravarty M, Satish Kumar N, Sajna K V and Kumara Swamy K C 2009 J. Chem. Sci. 121 23; (b) Kalek M, Johansson T, Jezowska M, Stawinski J 2010 Org. Lett. 12 4702; (c) Gomes F, Fadel A, Rabasso N 2012 J. Org. Chem. 775439

11. Selected recent applications: (a) Crioche J Meyer C and Cossy J 2013 Org. Lett. 15 1626; (b) Jose A Seetha Lakshmi K C Suresh E and Nair V 2013 Org. Lett. 15 1858; (c) Alcaide B, Almendros P Cembellin S Campo T M D and Fernández I 2013 Chem. Commun. 49 1282; (d) Deng Y and Backvall J E 2013 Angew. Chem. Int. Edn. 52 3217; (e) Gangadhararao G, Kotikalapudi R, Nagarjuna Reddy M and Kumara Swamy K C 2014 Beilstein J. Org. Chem. 10, 996

12. Gangadhararao G and Kumara Swamy K C 2014 Tetrahedron 702643

13. Only one report on such rearrangement (but no X-ray structural proof) is available prior to our study. See: Grachev M K, Nifant'ev E E 1989 Zh. Obshch. Khim. 59 1729; 1990 Chem. Abstr. 112 98658z

14. Perrin D D, Armarego W L F and Perrin D R 1986 Purification of Laboratory Chemicals Oxford, UK: Pergamon

15. (a) Gronnier C, Boissonnat G and Gagosz F 2013 Org. Lett. 15 4235; (b) Pisaneschi F, Sejberg J J P, Blain C, Wang H N, Aboagye E O and Spivey A C 2011 SYNLETT 0241

16. Davis A R, Dronsfield A T, Hazeldine R N and Taylor D R 1973 J. Chem. Soc., Perkin Trans. 1379

17. (a) Sheldrick G M 1997 SHELX-97 - A program for crystal structure solution and refinement, University of Göttingen; (b) Sheldrick G M 1996 SADABS, Siemens Area Detector Absorption Correction, University of Göttingen, Germany; (c) Sheldrick G M 1999 SHELXTL NT Crystal Structure Analysis Package, version 5.10; Bruker AXS, Analytical X-ray System: WI, USA

18. Keat R, Rycroft D S and Thomson D G 1980 J. Chem. Soc., Dalton Trans. 321

19. Kommana P, Kumaraswamy S, Vittal J J and Kumara Swamy K C 2002 Inorg. Chem. 412356

20. Corbridge D E C 2000 Phosphorus 2000: Chemistry, Biochemistry and Technology, 1st ed., Amsterdam: Elsevier, p. 69 (we have used Pauling electronegativity for this calculation) 